\title{
The effect of loading on position sense in the tongue
}

\author{
CYNTHIA GROVER and BRIAN CRASKE \\ Memorial University of Newfoundland, St. John's, Newfoundland, Canada
}

\begin{abstract}
The position sense of the tongue and aftereffects on the position sense subsequent to loading were directly investigated. Nine subjects judged the straightahead with the tongue before and after straining laterally with the tongue against the horizontal force induced by a 29.5-g weight. Two of these subjects also participated in the experiment after the surface of the tongue had been anesthetized. Prior loading of the tongue biased the subjects' subsequent judgment of the straightahead; following loading, the tongue was placed farther in the direction of the previous effort. The results are discussed with reference to postcontraction effects from experiments on loading the limb and eye.
\end{abstract}

We wished to investigate position sense in the tongue. The questions that interested us were whether the position of the tongue could be sensed, and whether there might be an aftereffect of loading the tongue. We wanted to compare the aftereffect, should it arise, with the effects of load on the limb and eye. The extent to which the processes of position sense differ in different kinds of movable organs also concerned us. Position sense for the eye is thought to be principally conveyed by the corollary discharge, while it is viewed as being a less crucial mechanism for limb position sense (see McCloskey, 1978). The tongue is a muscular joint-free hydrostat, in that it is an organ of fixed volume with a skeleton comprising muscle and connective tissue (see Smith \& Kier, 1989). It has a different environment, and it exhibits different properties from those of the eye and limb; and so the major mechanisms that could convey lingual position sense might differ from those that mainly convey eye or limb position.

The existence of position sense is extremely important for any organ operating in a spatial context. Without sensing the position of a limb or segment, we cannot direct it to a given place and so cannot act purposefully with it upon the environment. Logically, this argument must hold for the tongue also, and since vision cannot substitute for kinesthesis for the tongue, the existence of accurate position sense is crucial. Our capacity to produce

\footnotetext{
We would like to thank the departments of Psychology, Linguistics, and Medicine at Memorial University of Newfoundland, and the Natural Sciences and Engineering Research Council of Canada, for assistance with this project. We are grateful to Henry Manson, Anesthesiology, Memorial University of Newfoundland, for his advice on, and assistance with, all aspects of the anesthetic procedures. We would also like to thank anonymous referees for their comments on earlier versions of this manuscript. We are very grateful to our naive subjects, who submitted willingly to the somewhat undignified experimental procedures we required. Correspondence should be addressed to Cynthia Grover, Department of Psychology, Memorial University of Newfoundland, St. John's, Newfoundland A1B 3X9, Canada.
}

the sounds of speech reliably must rest firmly on our ability to sense the position of the tongue. This tenet has been recognized by numerous researchers in speech-related fields (e.g., Lowe, 1981; MacNeilage, 1970; Perkell, 1979; Sheldon \& Strange, 1982; Starkweather, 1983).

Originally, it was thought that the tongue did not have position sense (Goldscheider, 1898). The first study of position sense in the tongue was carried out by Carleton (1938). Her finding, that the tongue's position was not sensed well under anesthetic, was refuted by Weddell, Harpman, Lambley, and Young (1940), as well as by Adatia and Gehring (1971). Recently, Siegel and Hanlon (1983) conducted a distance estimation experiment with the tongue which implied very clearly that lingual kinesthesis was operative. In addition, anatomical investigators have consistently maintained that the spindles in the tongue muscles could relay the tongue's position (Bowman, 1971; Cooper, 1953; Langworthy, 1924; Tarkhan, 1936).

Nonetheless, the view that tongue position is not strongly perceptible, except perhaps via the lingual mucosa, remains widespread (e.g., Merton, 1964), perhaps because of papers on oral sensory deprivation in which the separate contributions to lingual position sense of elements other than skin could not definitively be estimated. Gammon, Smith, Daniloff, and Kim (1971), Scott and Ringel (1971), and Ringel and Steer (1963) could not judge the role of the muscles and corollary discharge in the sensing of tongue position, because they could not be certain of which elements that their nerve block had disabled. Putnam and Ringel (1976) did allot a role to the spindles in conveying position sense, but they inexplicably remarked that the resulting proprioceptive sensation was not available to consciousness.

The skin of the tongue is clearly highly sensitive and could signal tongue position, either on contact with structures, such as the teeth, or following deformation of the lingual mucosa-for example, with stretch of the tongue. These kinesthetic properties of the skin are of interest to us. Accordingly, we sought to discover what the skin's 
contribution to the tongue's sense of position might be by comparing performance during surface anesthesia of the tongue to performance without anesthetic.

We wished to use direct measurement to assess the sense of position of the tongue and to discover whether a bias could be induced in the judgment of tongue position similar to the biases observed in association with muscular strain for the eye, and under certain circumstances of strain in the limb. We use the term strain to mean effortful muscular work by a subject against (external) force. So, with respect to the lifting of a heavy parcel with one hand, we would say that the arm muscles strain upward against the downward force exerted by the weight.

In other studies of sensory systems, the aftereffect has been a useful tool in the attempt to infer underlying processes. We hoped that a study of the aftereffect of loading would provide insight into the spatial and sensory properties of a joint-free organ, the tongue. Comparison with aftereffects in other organs would be possible, since both the eyes and the limbs are known to exhibit good position sense under a range of conditions, but to exhibit marked differences under load.

The eye, like the tongue, has no joint, and so the potential sources of kinesthetic sensation are fewer than those available in the limb. Under load, judgments of eye position in error by as much as $90^{\circ}$ have occurred (Skavenski, Haddad, \& Steinman, 1972). Errors of this magnitude have never been observed in the loaded limb, even with muscle vibration. It is possible that the magnitude of the error for the eye is due to the lack of calibration of eye position for the amount of force exerted to turn the eye, or because it has no joint receptors. We were interested to know whether such large aftereffects might also be associated with loading the tongue. Like the eye, it has no joint; but like the limb, it is accustomed to bearing a variable load.

Given the fact that muscle is intimately involved in position sense, the evidence that muscular work influences perceived joint angle or judged distance (e.g., between fingers) is not immediately compelling. Under normal circumstances, the muscles must increase their tension to overcome a force-for example, gravity-or to maintain a limb's position, or to move the limb and a mass from one position to another. There is plenty of research to show that exerted force and adopted limb position are normally sensed accurately and independently (see Rymer \& D'Almeida, 1980), although the sense of effort may influence the perception of position (see McCloskey, 1981).

There is, nonetheless, some evidence to suggest that under special conditions muscular work can be associated with misjudgments of position. Misjudging the position of the arm may depend on the type of muscular work and on expectations about the work to be accomplished. Experiments that require the exertion of force but do not allow calibration of that force against position are rather unnatural. For example, the consistent misjudgments of finger position by Watson, Colebatch, and McCloskey's subjects (1984) may well have been due to the subjects' not knowing how far their fingers had moved when they exerted force against springs of various stiffness in the absence of vision. The subjects were allowed to check visually their finger positioning frequently, but it is not clear that they were allowed at any time to check visually the position of the fingertip while working against the springs. To the extent that position sense is a muscular phenomenon, it is not surprising that errors in judging position occur when subjects have not been given the chance to calibrate the tradeoff between afference due to force and that due to position.

The bias in sensing position appears to depend on the level of force previously exerted by the subject. The reliable misjudgment of position may well represent an attempt to deduce the ultimate position, using as a relevant, but misleading, parameter the force exerted to move the limb into position. Thus the estimate of the work to be accomplished could be argued to bias the subjects' sense of the extent of movement, and ultimately of position. Similar effects have been noted in studies of the effect of load on the sensed extent of movement (Roland \& Ladegaard-Pedersen, 1977; Rymer \& D'Almeida, 1980).

Other experiments have also shown that the use of muscle and the direction of muscular work bias the sensed position of the limb and eye. Thus the limb (or eye) is perceived to occupy a position farther in the direction opposite the direction of the effort than is correct.

Several types of experimental procedure have been employed in studies of the effect of muscular contraction on judged position. In one case, the limb or eye makes a large voluntary movement. Judgments of its ultimate position after termination of movement reveal that the limb is perceived to be farther in the direction opposite the direction of movement than is correct (Craske \& Crawshaw, 1974; Craske, Crawshaw, \& Heron, 1975; Hoff \& Schilder, 1925; Howard \& Templeton, 1966; Park, 1969; Slinger \& Horsley, 1906). Gregory, Morgan, and Proske (1988) have suggested that the direction of the error in judging arm position depends upon whether the previously contracted muscle is a flexor (biceps) or an extensor (triceps). More research on different limbs must be done to address the relationship between the direction of positional error and the functional role of the muscle for this finding to be viewed as generally valid.

In the second type of procedure, which involves what we call strain, a limb position is maintained with effort against a force. Limb position is indicated during the effortful work as being in the direction opposite the one in which the limb is straining (McCloskey, 1973). Perhaps most directly relevant to investigation involving the tongue is the large, but directionally unspecified, bias in sensed eye position during loading that was observed by Skavenski et al. (1972).

Related positional biases are displayed in the illusion of impact and the series effects outlined by Hollingworth (1909). Howard and Templeton (1966) have speculated that persistence of muscular tension, sensory adaptation, and/or central processes might each have a role to play. More recently, spindle response facilitation, motoneuronal 
pool potentiation, and muscle fiber twitch potentiation have been proposed as contributors to the bias (Gregory et al., 1988; Hutton, Enoka, \& Suzuki, 1984).

A muscle that has just borne a load continues to discharge (Hutton, Smith, \& Eldred, 1973). It is not fully clear how the accompanying misinterpretation of position arises: whether the spindles alone are affected, or whether the corollary discharge associated with willed movements after relief from the load might also contribute to the bias.

The evidence for these biases is drawn from research on the eyes and limbs; from the current state of knowledge about position sense, the inference to be made is that these effects arise at least in part from biases in information from the muscles. Consequently, to the extent that the tongue uses kinesthetic mechanisms that are based on muscle, we would expect to find normally good position sense in the tongue that can be biased by previous loading.

Furthermore, by examining the effects of loading on position sense in the normal and surface-anesthetized tongue, we should have evidence concerning the role of the cutaneous sheet as a sensory source of lingual kinesthesis. Given the articulatory imprecision that is evident during topical lingual anesthesia (Scott \& Ringel, 1971), we wondered whether normal tactile sensation would reduce any aftereffect by overwhelming the muscular proprioceptive sensations that might contribute to it.

An experiment was designed to answer these questions. We hypothesized that:

1. Straining the tongue against a force produces errors in judging the straightahead with the tongue. Subjects should tend to place the tongue in the direction of previous effort.

2. The skin of the tongue may contribute important information about tongue position, in which case errors in tongue positioning should be smaller when the lingual mucosa are not anesthetized.

In the rest of this paper, we describe the method, apparatus, and procedures with which we obtained, analyzed, and interpreted our data.

\section{METHOD}

\section{Subjects}

Five female and 5 male adults were informed of our experimental procedures and consented to participate. The 8 naive paid subjects completed all nonanesthetic conditions, 1 unpaid subject (C.G.) performed in all four conditions, and the other unpaid subject (B.C.) took part in three experimental conditions. The naive subjects were not aware of the experimental hypotheses.

\footnotetext{
Materials

A headrest incorporating a padded nose support, chinrest, and head strap was used to keep the subject's head in position during the experiment (see Figure 1). The headrest supported a flat plastic sheet, $75 \mathrm{~mm}$ wide and $39 \mathrm{~mm}$ deep in a horizontal plane. This scale abutted the subject's lower lip. It was marked in degrees of arc for later use in measuring tongue position from videotape. A range of $20^{\circ}$ of lingual angle, centered about the mid-saggital plane, was represented. Following Kahane (1982), lingual angle was calculated assuming a tongue length from root to the lower teeth of $80 \pm 8 \mathrm{~mm}$.
}

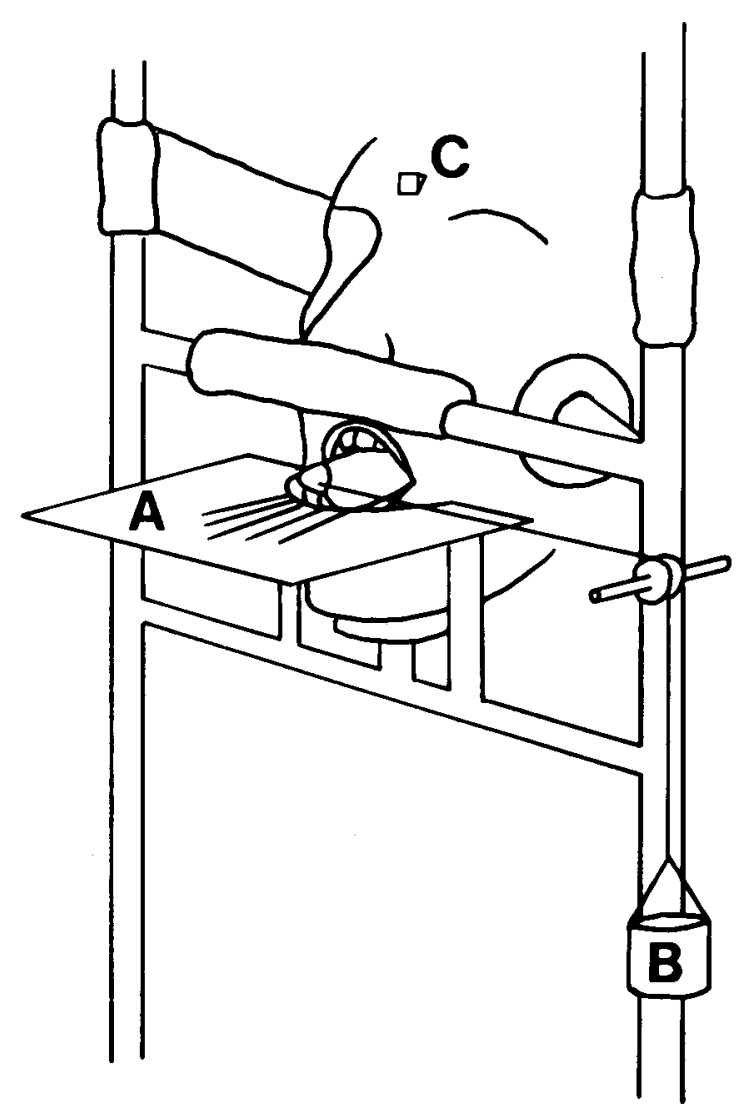

Figure 1. The headrest used in Experiment 1 (based on a photograph by Jack Martin). (A) Scale marked in degrees. (B) Mass suspended over a pulley. (C) Mirror on forehead.

A brass mass of $29.5 \mathrm{~g}$ was attached with a piece of lightweight nylon thread to a truncated cap $17 \mathrm{~mm}$ long through a small hole drilled in its side, $3 \mathrm{~mm}$ from the base. This mass hung freely when suspended over a small pulley on the headrest.

A 2-cm-square front surface mirror was attached to a wedge of adhesive-backed sponge. This was fixed to the center of the subject's forehead, where it remained throughout the experiment. It reflected an arrow of light onto a section of wall marked in degrees of arc relative to the head. The experimenter maintained the subject's head in position by ensuring that the reflected light remained within a pair of demarcations on the wall denoting $0.5^{\circ}$ of head rotation in a horizontal plane.

The fiducial mark on the tongue was a semipermanent dark dot made with a nontoxic felt pen and a flour paste dot overpainted on this with a Size 00 paintbrush. This coincident pair of dots was located at the center of the upper surface of the tongue tip and served as the visible mark for the video camera. Approximately 4 parts all-purpose flour to 3 parts water produced a nontoxic, painless, virtually tasteless, visible paste.

A standard low-light laboratory camera was positioned in the subject's midsaggital plane so that tongue movement against the background of the scale could be videotaped with a magnification of $5: 1$. The position of the tongue in half degrees of lingual angle was easily read from the magnified image of the tongue.

\section{Procedure}

The logic of the experiment was the following. Judgments of tongue position before and after loading were to be compared. Ac- 
cordingly, three blocks of control trials (before loading) and one block of experimental trials (after loading) were conducted. In order to ensure that effects associated with the experimental trials were indeed due to the horizontal load and not merely to the exertion of effort required to simply protrude the tongue straight ahead, judgments of tongue position before and after simple tongue protrusion were also made. Thus, in the first and third blocks of (basic control) trials, the subject judged tongue position. In the second (protrusion control) block of trials, the subject protruded the tongue for $30 \mathrm{sec}$ before judging tongue position. The order of trials follows:

Control trials. (1) Judgment of the straightahead position with the tongue, followed by a rest. (2) Protrusion of the tongue straightahead for $30 \mathrm{sec}$, followed by judgment of the straightahead and then a rest. (3) Judgment of the straightahead position with the tongue, followed by a rest.

Experimental trials. Protrusion of the tongue straight ahead, the position being maintained against a horizontally applied load for $30 \mathrm{sec}$, followed by judgment of the straightahead (no load) and then a rest.

Each experimental condition comprised 4 blocks of 10 trials in the above order. The order of blocks did not vary. The subjects participated in the experiment one at a time.

Once the headrest and the accompanying apparatus had been adjusted so that the subject was comfortable, the subject left the headrest, and videotaping commenced. The experimenter made a dark dot with the felt marker at the center of the upper surface of the subject's tongue tip. This dot did not fade during the course of the experiment. Before each block of trials, the experimenter placed a dot of flour paste on top of it.

The subject entered the headrest, and the experimenter fastened the headstrap around the subject's head. The subject shut the eyes at this point and kept them shut throughout all trials. The subject opened the mouth so that the lower jaw was stationed on the chinrest and the nose on the nose bar. The subject maintained this position throughout each block of trials. The experimenter placed her hands on the subject's head to keep it in position. Then she instructed the subject to judge when the tongue was felt to be straight ahead, using the method of adjustment. When the experimenter called out "left," for example, the subject protruded the tongue from the left side of the mouth, moved the tongue to the right until satisfied that it was straight ahead, and then dropped it gently onto the scale and knocked on the table to indicate that the tongue was straight ahead and on the scale. Then the subject retracted the tongue and the next trial began.

Trials starting from the two sides of the mouth alternated, for a total of 10 trials per block. The trials each took about $3 \mathrm{sec}$, on the average. After completing a block of trials, the subject exited the headrest, wiped the flour paste off the tongue, and rested.

Blocks 1 and 3 proceeded in this fashion. In Block 2, the subject protruded the tongue straight ahead for $30 \mathrm{sec}$, taking care not to touch it to the scale. The experimenter then painted a flour paste dot on top of the dark dot on the subject's tongue, and the subject judged the straightahead 10 times, as before. The subject then left the headrest and wiped the flour paste off the tongue. These 10 trials served as a second type of control (protrusion).

Before Block 4 (the experimental block), the subject sucked the cap onto the tongue tip and entered the headrest. Then the subject protruded the tongue, and the mass was gently released over the pulley. The subject's task was to maintain the tongue straight ahead for $30 \mathrm{sec}$ without letting it rest on the scale. Once the $30 \mathrm{sec}$ had elapsed, the experimenter released the suction, removed the cap from the subject's tongue, and painted on a flour dot. The subject judged the straightahead 10 times and then left the headrest.
All conditions were composed of these four types of trials. The various conditions were the following: performance with anesthesia of the mucosa, performance without anesthesia, and performance following a weight pulling the tongue to the right (eliciting straining with the tongue to the left) and following a weight pulling the tongue to the left (eliciting straining with the tongue to the right). The subjects underwent the various conditions in different orders, with at least a day between participation in any two conditions.

Two subjects took part in the anesthetic conditions in an earlier version of this experiment. These conditions were similar to the nonanesthetic conditions described above. The procedural differences were as follows. Anesthesia of the lingual mucosa was induced before any trials were conducted. The mirror on the forehead and the dark dot on the tongue were not used. In their place, head position was monitored by recording the position of a flour paste stripe on the lower lip directly before every block of trials. Also, the flour paste dot was repainted in the same place on the tongue, each time using landmarks such as the central sulcus. Last, trials occurred at timed 3-sec intervals, and the subjects did not need to knock to indicate when they felt the tongue to be straight ahead.

A dosage (60 mg for 1 subject and $90 \mathrm{mg}$ for the other) of a $4 \%$ solution of Xylocaine was administered to the upper and lower surfaces of each subject's tongue. Testing ensured that this was sufficient to eliminate sensations of contact and pressure between the tongue and objects placed in the mouth.

\section{Measurement and Analysis}

The videotaped sessions were played back on television. Measurement of the straightahead of the subject's head in the anesthetic condition was made by stopping the videotape when contact between the lower lip and the scale was seen to occur, and then reading from the image of the scale the location of the center of the stripe on the lower lip to a half degree of arc. The standard deviation of the error in these measurements (due to the assumption of equal tongue length across subjects) should be $0.1^{\circ}$ per $1^{\circ}$ of angle of the tongue from the center of the mouth in the horizontal plane.

For the anesthetic condition, judgments with the tongue of the straightahead were measured by stopping the videotape when the tongue tip was seen to protrude to its farthest point and reading to a half degree of arc the location of the center of the dot on the tongue tip with respect to the markings on the scale. Changes from an arbitrary zero indicated by the lower lip marker were subtracted from the measure of tongue position for each trial in a block.

For the nonanesthetic condition, head position was kept constant, so the position of the flour paste dot was read directly from the scale on the videotape when the subject's knock was heard. In cases in which the flour paste had been inadvertently smeared, it was often possible to discern the position of the dark dot, and this was then used for measurement instead.

In total, there were 828 measurements of the tongue's position to analyze. One subject's data had to be discarded due to her failure to follow instructions. Another subject was not available for one session with the anesthetist for reasons unrelated to the experiment.

The occasional trial could not be measured, due to failure of the tongue to contact the scale or due to smearing of the flour paste. The other trial in the left-right pair was then also removed from analysis. For six data sets, there are thus 38 instead of 40 trials per subject.

A reliability check on data measurement was conducted, using an observer naive with respect to the purpose of the experiment. The correlation between observers' measurements gave $r=.93$.

The statistical treatment consisted of repeated measures multiple regression. The variance due to subjects was removed from the 
Table 1

Mean Deviations from $0^{\circ}$ (Degrees of Lingual Angle)

\begin{tabular}{|c|c|c|c|c|c|c|c|c|}
\hline \multirow[b]{4}{*}{ Block } & \multicolumn{8}{|c|}{ Direction of Effort } \\
\hline & \multicolumn{4}{|c|}{ After Straining Right } & \multicolumn{4}{|c|}{ After Straining Left } \\
\hline & \multicolumn{2}{|c|}{ No Anesthetic } & \multicolumn{2}{|c|}{ Anesthetic } & \multicolumn{2}{|c|}{ No Anesthetic } & \multicolumn{2}{|c|}{ Anesthetic } \\
\hline & $M$ & $S D$ & $M$ & $S D$ & $M$ & $S D$ & $M$ & $S D$ \\
\hline $\mathrm{Cl}$ & -0.81 & 2.19 & 0.20 & 0.35 & -0.11 & 2.30 & 0.73 & 1.33 \\
\hline $\mathbf{P}$ & -0.93 & 2.87 & 0.40 & 0.40 & -0.60 & 2.89 & 0.35 & 1.42 \\
\hline C3 & -0.34 & 3.06 & 0.90 & 0.46 & -0.50 & 3.25 & 0.83 & 2.04 \\
\hline $\mathbf{L}$ & 0.39 & 3.03 & 1.70 & 0.59 & -1.22 & 3.16 & -0.53 & 1.01 \\
\hline
\end{tabular}

Note $-\mathrm{Cl}=$ first control block; $\mathrm{P}=$ protrusion control block; $\mathrm{C} 3=$ third control block; $\mathrm{L}=$ trials after loading.

regression equations before the effects due to treatment were examined.

\section{RESULTS}

First, we considered only the data from the control trials of the nonanesthetic condition. A repeated measures multiple regression analysis was carried out on the control trial data. Judgment of the straightahead during the basic control trials did not differ significantly from judgments after protruding the tongue straight ahead for $30 \mathrm{sec}$ : For the nonanesthetic condition, $B$ (standardized Beta) $=0.05$, $F(1,520)=2.24, p=.13$, and for the anesthetic condition, $B=0.12, F(1,87)=3.33, p=.07$ (after removal of the variance due to subjects). The mean deviation and standard deviation from the objective straightahead were calculated for each block of trials. These statistics are presented in Table 1.

Consequently, we collapsed the protrusion and basic control data in order to compare judgment of the straightahead after loading the tongue with judgment in all the control trials.

We assumed that the subjects would select a tongue position as being straight ahead and continue to select that position, relative to the scale. The scale was placed with the $0^{\circ}$ mark approximately straight ahead of the subject (within $5^{\circ}$ of the midsaggital plane of the subject's head). Although the scale's zero is, strictly speaking, arbitrary, the consistent judgment of straight ahead as being centered at $-0.55^{\circ}$ (left of center) with a standard deviation of $2.8^{\circ}$ within that central $5^{\circ}$ implies that subjects know where straight ahead of the tongue is.

To correct for differences in placement of the scale for each subject, we normalized the data as follows. Within each subject's three blocks of control data in the nonanesthetic condition, the mean judged straightahead was set to zero, and the standard deviation was then calculated across all subjects' normalized control data. Subjects do tend to choose narrowly a position as being straight ahead, for the resulting standard deviation is $1.6^{\circ}$. We conclude that a sense of the tongue's position exists and is reliable and fairly accurate.

Horizontal strain by the tongue altered the sensed horizontal position of the tongue tip. One subject's data is shown by way of example in Figure 2. After straining against the weight for $30 \mathrm{sec}$, the subject placed the tongue farther in the direction of previous effort when judging the straightahead. For most subjects, the tongue was perceived to occupy a position farther in the direction opposite that of the previous effort than was objectively the case. Thus the tongue was placed farther to the left after straining leftward and vice versa. This trend is clear in Figure 3: For the nonanesthetic condition after straining with the tongue to the right, $B=0.16, F(1,342)=19.1$, $p<.0001$; and after straining to the left, $B=-0.13$, $F(1,346)=16.7, p=.0001$ (after removing the variance due to the subjects).

For the anesthetic data, the same effect occurred. After straining rightward with the tongue, $B=0.72, F(1,38)$ $=40.6, p<.0001$; after straining leftward, $B=-0.32$, $F(1,77)=33.4, p<.0001$. These findings support the hypothesis that effortful muscular work biases the sensed position of the tongue, in such a way that the tongue is perceived to be farther in the direction opposite to the ef-

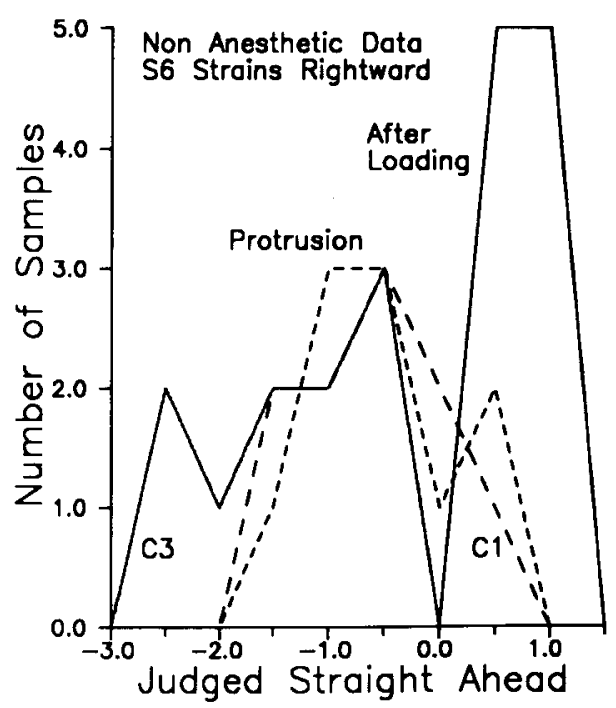

Figure 2. Graph of Subject 6's data (nonanesthetic condition: straining rightward with the tongue). $\mathrm{Cl}=$ first block of control trials; $\mathrm{C3}=$ third block of control trials. 


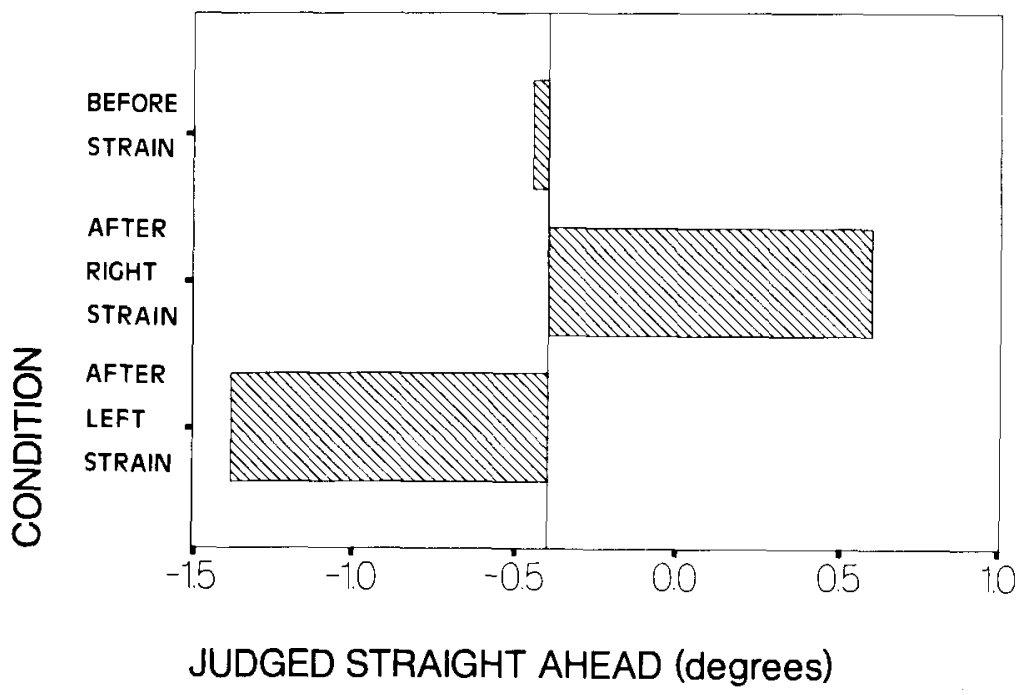

Figure 3. Judgments of the straightahead by 9 subjects with the nonanesthetized tongue after loading. The three control conditions (before strain) are represented in the uppermost bar of the figure $(n=530)$. Judgment after straining with the tongue is shown by the bottom bars ( $n=178$ for each). The scale was placed with the arbitrary $0^{\circ}$ mark approximately straight ahead of the subject (within $5^{\circ}$ of the midsaggital plane of the subject's head). The reference line is placed at the mean judged straightahead across all data.

fort than is true. This result conforms to previous data for other kinesthetic systems.

An inspection of the decay of the effect of strain was carried out. There was no statistically significant linear trend over time within the nonanesthetic data for either direction of effort: After strain with the tongue rightward, $F(1,78)=0.63, p>.05$; and after straining leftward, $F(1,80)=1.45, p>.05$ (see Figure 4). The weakness of the trends is due to the large variance in the data (even

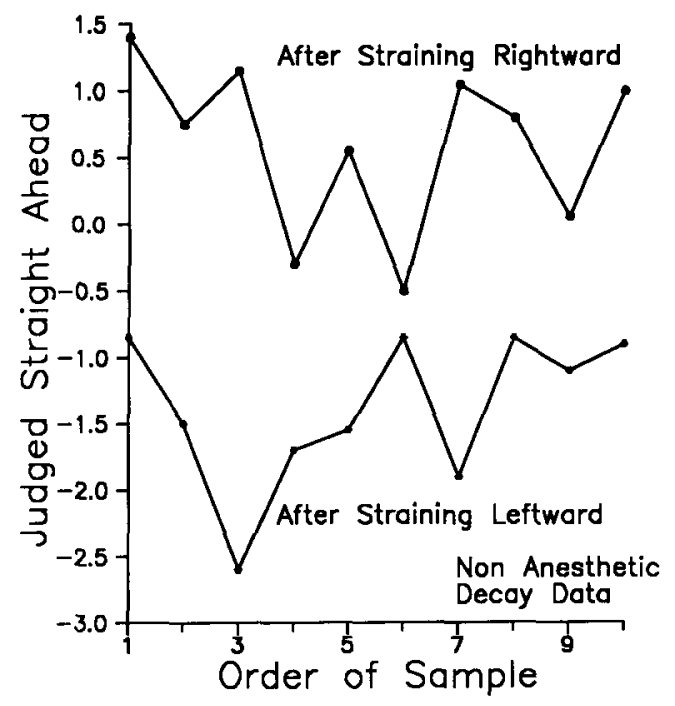

Figure 4. The decay of the effect of loading on judgment of the straightahead for all subjects in the nonanesthetic condition. The 10 judgments per subject were made over approximately $30 \mathrm{sec}$ after the load had been removed from the tongue. after removal of variance due to subjects). Nonetheless, the trends in the two directions of aftereffect were significantly different $[F(1,174)=18.7, p<.01]$. As the time after the effort increased, the subjects' judgments of the straightahead tended to approach from opposite directions the average straightahead position adopted during the control trials.

We expected that anesthesia of the mucosa would have little effect on kinesthesis in the tongue, for the work against the weight should involve the muscles, and not the skin of the tongue. Our results are inconclusive with respect to this question. A comparison of the effect of straining with and without anesthetic for the 2 subjects whose tongues were anesthetized produced conflicting results. No reliable trend was apparent.

Overall, the aftereffect of muscular work is similar to that observed elsewhere; from the previously judged straightahead, there was a mean deviation of about $1^{\circ}$ in the direction of previous effort.

\section{CONCLUSION}

The tongue displays position sense. The placement of the tongue in the prestrain trials was straight ahead. Effortful muscular work biases the sense of the tongue's position. The registered straightahead for the tongue is shifted in the direction of the previous effort, and this bias is not reliably affected by anesthesia of the mucosa.

The direction and size of the bias that we elicited by loading the tongue square with those mentioned in previous research on muscular work with other organs. Our mean shift in positioning the tongue following strain with it is about $1^{\circ}$, whereas Craske et al. (1975) found a mean shift of $0.84^{\circ}$ in centering the eye after maintaining the 
eyes to one side. For comparison, the limits of positioning the eye and limb in the dark are around $3^{\circ}$ (Merton, 1961). Clearly, the bias induced by loading the tongue is similar to the work-induced biases of limb and eye position, if judgments of position are taken after loading the tongue.

Larger biases have been elicited during loading $\left(4.2^{\circ}\right.$ for the forearm, calculated from McCloskey, 1973, and up to $90^{\circ}$ for the eye, in Skavenski et al., 1972), and so one could speculate that eliciting judgments about tongue position during loading might produce a larger bias as well.

Calibration of position against the force exerted to bear the load is a prerequisite to sensing position accurately, and may be partly responsible for the larger errors observed by McCloskey (1973) and Skavenski et al. (1972) during loading. In the case of the tongue, contact with structures of the mouth such as the teeth or lips, after the load has been removed, could have provided information that allowed recalibration of position sense. Contact with an immovable familiar object informs one about what muscle lengths, corollary discharges, and tendon states must be achieved to attain a certain position. It is all the more surprising that a measurable bias in tongue position persists during use of the method of adjustment, which allows contact with the sides of the mouth before judgment of the straightahead.

The brain may use afference from the tongue muscles and tendons to infer lingual position. This deduction seems reasonable, since the bias in tongue positioning is observed to have the same direction as that observed under similar circumstances in the limb and eye.

Our results are congruent with results from recent research on postcontraction (Gregory et al., 1988; Hutton et al., 1984). The bias that we observed could be viewed as part of the postcontraction effect. The observations of Gregory et al. (1988) indicate that, after contraction, the spindles continue to supply afference. If that afference is interpreted wrongly, thus providing an inaccurate reading of current position, our results will be predicted.

We think that it is also very likely that the corollary discharge is a prime contributor to the observed bias. We assume that the main protruder of the tongue in our experiments was the genioglossus, and that the intrinsic muscles principally worked against the load (see Lowe, 1981). In agreement with Hutton et al. (1984) and Gregory et al. (1988), we assume that there is a motor postdischarge in the intrinsic muscles on the previously stiff side of the tongue, the side that principally bore the load. This results in movement of the tongue in the direction of the previous effort, movement of which the subject is perhaps unaware. Although the spindles on the flaccid side of the tongue fire to signal stretch, there is no relevant corollary discharge, because the movement due to motor postdischarge is not willed. If we assume further that this postcontraction discharge continues as the subject moves the tongue from the side of the mouth to the straightahead, then the corollary discharge associated with this intended movement will continue to underrepresent the extent of actual movement in the direction opposite that of the previous effort.

The spindles on the previously stiff side of the tongue could contribute to the observed bias if their signaling of stretch were affected by the muscular work. This could occur when the tongue was moving toward the straightahead from the side that remained more flaccid throughout the experiment. Only in this context (that is, for positional errors made after movement from one side of the mouth) could the spindles possibly be the sole source of the bias. We did not see clear evidence of a stronger aftereffect in association with movements from the side of the body where the load had been placed.

We presumed that the skin would not contribute to the bias, because the work was muscular. If anything, information from the unanesthetized mucosa of the tongue should have provided veridical information based on sensed deformation of the tongue surface, which might have reduced the bias in judged straightahead, relative to the anesthetic condition. Our presumption was confirmed. The biases in the judged straightahead with and without anesthesia of the tongue surface had similar magnitudes and identical directions. In the presence of misleading kinesthetic afference and an unwilled motor discharge, the tactile afference from the unanesthetized surface of the tongue generally fails to correct for the bias. This afference either fails to provide clues about tongue position, or is not attended to in this nonspeech task.

Our results provide evidence that a hydrostatic organ without a joint operates in some respects as do the jointed bony segments to which most skeletal muscles are attached. Not only can the muscles of the tongue move the tongue about its point of attachment, as do muscles that are attached to bone, they can also, by their contraction, change the tongue's shape, preserving as constant only the volume of fluid in the tongue, and not its length (Smith \& Kier, 1989). Kinesthetic interpretation of changes in tongue muscle length should then be less straightforward than that of skeletal muscle length, since not only positional change of the tongue tip, but also change in tongue shape can be associated with tongue muscle shortening. Hydrostatic systems such as the tongue or the elephant's trunk have many more degrees of freedom of movement than do bony segments, because of their lack of rigid support. It is of interest to discover that their basic principles of operation seem nonetheless to be similar to those of jointed structures.

\section{REFERENCES}

Adatia, A. K., \& Gehring, E. N. (1971). Proprioceptive innervation of the tongue. Joumal of Anatomy, 110, 215-220.

BoWmaN, J. (1971). The muscle spindle and neural control of the tongue: Implications for speech (pp. 116-148). Springfield, IL: Thomas. 
CARLETON, A. (1938). Observations on the problem of the proprioceptive innervation of the tongue. Journal of Anatomy, 72, 502-507.

COOPER, S. (1953). Muscle spindles in the intrinsic muscles of the human tongue. Journal of Physiology, 122, 193-202.

Craske, B., CRawshaw, M. (1974). Differential errors of kinesthesis produced by previous limb positions. Journal of Motor Behavior, 6, 273-278.

Craske, B., Crawshaw, M., \& Heron, P. (1975). Disturbance of the oculomotor system due to lateral fixation. Quarterly Journal of Experimental Psychology, 27, 459-465.

Gammon, S., Smith, P., Daniloff, R., \& Kim, C. (1971). Articulation and stress/juncture production under oral anesthetization and masking. Journal of Speech \& Hearing Research, 14, 271-282.

GoldSChEIDER, A. (1898). Über den Muskelsinn und die Theorie der Ataxie [On muscle sense and the theory of ataxia]. Gesammelte $A b$ handlungen: Band 2. Physiologie des Muskelsinnes (pp. 40-43). Leipzig: Johann Ambrosius Barth.

Gregory, J. E., Morgan, D. L., \& Proske, U. (1988). Aftereffects in the response of cat muscle spindles and errors of limb position sense in man. Journal of Neurophysiology, 59, 1220-1230.

HoFF, H., \& SCHILDER, P. (1925). Über Lagebeharrung. Monatsschrift für Psychiatrie und Neurologie, 58, 257-264.

Hollingworth, H. L. (1909). The inaccuracy of movement. Archives of Psychology, 13, 1-87.

HowARD, I., \& TEMPLETON, W. (1966). Human spatial orientation. London: Wiley.

Hutton, R. S., Enoka, R. M., \& Suzuki, S. (1984). Activation history and constant errors in human force production. Brain Research, 307, 344-346.

Hutton, R. S., SMIth, J. L., Eldred, E. (1973). Postcontraction sensory discharge from muscle and its source. Joumal of Neurophysiology, 36, 1090-1103.

KAHANE, J. (1982). Anatomy and physiology of the organs of the peripheral speech mechanism. In N. Lass, L. McReynolds, J. Northern, \& D. Yoder (Eds.), Speech, language and hearing: Vol. 1. Normal processes (pp. 109-155). Philadelphia: W. B. Saunders.

LANGWORTHY, $O$. (1924). A study of the innervation of the tongue musculature with particular reference to the proprioceptive mechanism. Journal of Comparative Neurology, 36, 273-297.

Lowe, A. A. (1981). The neural regulation of tongue movements. Progress in Neurobiology, 15, 295-344.

MacNeilage, P. (1970). Motor control of serial ordering of speech Psychological Review, 77, 182-196.

MCCloskey, D. I. (1973). Differences between the sense of movement and position shown by the effects of loading and vibration of muscles in man. Brain Research, 61, 119-131.

McCloskey, D. I. (1978), Kinesthetic sensibility. Physiological Reviews, 58, 763-820.

MCCloskey, D. I. (1981). Corollary discharges: Motor commands and perception. In V. B. Brooks (Ed.), Handbook of physiology: Sec. I. The nervous system: Vol. 2. Motor control (pp. 1415-1447). Bethesda, MD: American Physiological Society.
Merton, P. A. (1961), The accuracy of directing the eyes and the hand in the dark. Journal of Physiology, 156, 555-577.

Merton, P. A. (1964). Human position sense and sense of effort. Symposia of the Society for Experimental Biology, 18, 387-400.

PARK, J.N. (1969). Displacement of apparent straight ahead as an aftereffect of deviation of the eyes from normal position. Perceptual \& Motor Skills, 28, 591-597.

Perkell, J. (1979). On the use of sensory feedback: An interpretation of compensatory articulation experiments. Proceedings of the Ninth International Congress of Phonetic Sciences, 2, 358-364.

Putnam, A., \& Ringel, R. (1976). A cineradiographic study of articulation in two talkers with temporarily induced oral sensory deprivation. Journal of Speech \& Hearing Research, 19, 247-266.

Ringel, R., \& STEER, M. (1963). Some effects of tactile and auditory alterations on speech output. Journal of Speech \& Hearing Research, 6, 369-378.

Roland, P. E., \& LAdegaArd-Pedersen, H. (1977). Sensations of tension and kinaesthesia from musculotendinous receptors in man: Evidence for a muscular sense and a sense of effort. Brain, 100, 671-692.

RYmer, W. Z., D'AlmeidA, A. (1980). Joint position sense: The effects of muscle contraction. Brain, 103, 1-22.

SCOTT, C., \& RINGEL, R. (1971). Articulation without oral sensory control. Journal of Speech \& Hearing Research, 14, 804-818.

Sheldon, A., Strange, W. (1982). The acquisition of $/ \mathrm{r} /$ and $/ \mathrm{l} /$ by Japanese learners of English: Evidence that speech production can precede speech perception. Applied Psycholinguistics, 3, 243-261.

SiEgEL, G., \& HaNLON, J. (1983). Magnitude estimation of oral cavity distances. Journal of Speech \& Hearing Research, 26, 574-578.

Skavenski, A. A., Haddad, G., \& Steinman, R. M. (1972). The extraretinal signal for the visual perception of direction. Perception \& Psychophysics, 11, 287-290.

SLINGER, R. T., \& HORSLEY, V. (1906). Upon the orientation of points in space by the muscular, arthrodial, and tactile senses of the upper limbs in normal individuals and in blind persons. Brain, 29, 1-27.

SMITH, K. K., \& KIER, W. S. (1989). Trunks, tongues, and tentacles: Moving with skeletons of muscle. American Scientist, 77, 29-35.

Starkweather, C. W. (1983). Speech and language: Principles and processes of behavior change (pp. 174-192). Englewood Cliffs, NJ: Prentice-Hall.

TARKHAN, A. (1936). Ein experimenteller Beitrag zur Kenntnis der proprioceptiven Innervation der Zunge [An experimental paper on the question of the proprioceptive innervation of the tongue]. Zeitschrift für Anatomie und Entwicklungsgeschichte, 105, 349-358.

Watson, J. D. G., Colebatch, J. G., \& McCloskey, D. I. (1984). Effects of externally imposed elastic loads on the ability to estimate position and force. Behavioural Brain Research, 13, 267-271.

Weddell, G., Harpman, J. A., Lambley, D. G., \& Young, L. (1940). The innervation of the musculature of the tongue. Journal of Anatomy, 74, 255-266.

(Manuscript received May 25, 1990 ; revision accepted for publication February 20, 1991.) 